\title{
Sialylated Carbohydrates as Inhinitors of Coronavirus Infection
}

\author{
Jesus' Christus, Michael Arden Madson \\ Research and Development, Bio Logistics Limited Liability Company, Ames Iowa, United States of America \\ Email address: \\ mmadson53@earthlinknet (M. A. Madson)

\section{To cite this article:} \\ Jesus' Christus, Michael Arden Madson. Sialylated Carbohydrates as Inhinitors of Coronavirus Infection. American Journal of Biomedical \\ and Life Sciences. Vol. 9, No. 1, 2021, pp. 20-28. doi: 10.11648/j.ajbls.20210901.13
}

Received: November 23, 2020; Accepted: December 14, 2020; Published: January 22, 2021

\begin{abstract}
COVID-19 is pandemic. Neuraminidase ị central to both infection from the virus and is involved in the cell's endothelial cell rolling, as an example. Inhibition of glycosidases are known to include anhydro ald(ket)itols. An L-1,5anhydro fucitol substituted by a derivatized (dihydrido) sulfo hydrate has been synthesized from bovine thyroglobulin N-linked oligosaccharide dipeptide. Also, 2,5-anhydro 1,6 di-(hydrido) di-phospho dihydrate mannitol (glucitol) has been prepared. Both include a treatment with $\mathrm{NaBH} 4$ in $\mathrm{NH}_{4} \mathrm{OH}$. Here evidence is presented on 2,6-anhydro $\mathrm{N}$-acetamido neuraminitol under similar reaction conditions using Kappa casein and bovine submaxilary mucin (bsm). It is hoped to use these reaction conditions and apply it to bovine milk. It may be possible to synthesize 2,6-anhydro N-acetamido neuraminitol in two steps from bovine milk. Then treatment costs can be afforded by those who are financially compromised. Conditions used are 8 hours at ambient temperature in a capped or un-capped reaction vial. These glycoproteins were treated with PNGase-F which could contain peptidase activity that acts in appreciable quantities in the large excesses of PNGase-F used here. Then the effluent from an $\mathrm{NH}_{4}{ }^{+}$form cation exchange cartridge to which it was bound, after $\mathrm{H}_{2} \mathrm{O}$ wash, were eluted with $\mathrm{NH}_{4} \mathrm{OH}$ and partially evaporated to remove excess base. The reaction products were stored frozen prior to analysis by a single quadrupole mass spectrometer, AQA, or a triple quadrupole mass spectrometer, API 2000. Fetuin was treated in the same manner but was used only as a standard and not included as se a starting material. Hope is in the transfer of these protocols to the preparation pf 2,6-anhydro N-acetamido neuraminitol. It may act as a two-pronged attack on COVID-19 infection. Previous work suggests that the purification of 2,6-anhydro $\mathrm{N}$-acetamido neuraminitol is not trivial.
\end{abstract}

Keywords: COVID-19 Potential Infection Inhibitor, Glycoprotein, $\mathrm{NaBH}_{4} / \mathrm{NH}_{4} \mathrm{OH}, \mathrm{N}$, O-linked Oligosaccharide Dipeptide, Mass Spectrometry

\section{Introduction}

Coronavirus, COVID-19, is now pandemic. There are more than $11,400,796$ cases of coronavirus in the US and more than 249,000 deaths in the US alone. There are lock downs in order to limit infection rates. Clearly, the time to address infection by COVID-19 is immediate.

In the avian form of the virus, sialic acid has been shown to mediate hemagglutination by neuraminidase pretreatment, with no activity. [1] Sialic acid has also been postulated to mediate Mycobacterium lepramatous infection by binding Toll-Like Receptor 2 (TLR-2). [2] Neuraminidase, sialic acid glycoside hydrolyzing enzyme, is required to activate the causative agent from the organism Vibiro cholera, causing cholera. Hemaglutinaiton by corona virus can be inhibited by sialic acid containing gangliosides [3] Competitive inhibitors that mask the sialic acid mediated binding activity can be inactivated by neuraminidase treatment. [4] Sialic acid is a known receptor determinant for coronavirus. [4] An inhibitor of these phenomena would be highly desirable. In this work such a potential inhibitor may be prepared. It is the 2,6- Nacetamido neuraminitol prepared from bovine milk via novel chemistry that has been used to prepare 1,5 anhydro L-fucitol derivative and 2,5 anhydro 1,6 di- (hydrido) di-phospho dihydrate mannitolfrom bcmanana extract. [5, 6] Anhydro sugars have been shown to inhibit an $\alpha$ mannosidase. [7] Preparation of anhydro sugars have been shown to be present in the depolymerization of heparin. [8] And the anhydro sugar class, the deoxy nojirimycins, have been shown to be potent inhibitors of glycosidases. [9] It is not known whether 
there are $\mathrm{N}$-acetamido neuraminyl group receptors on the coronavirus itself that bind to an NANA group on the surface of the endothelial cell or that a coronavirus neuramidase cleaves a host cell $\mathrm{N}$-acetamido neuraminyl. group. The 2,6 anhydro N-acetamido neuraminitol could inhibit both mechanisms of coronavirus infection of the host. Here, evidence is presented to support the preparation of 2,6anhydro $\mathrm{N}$-acetamido neuraminitol in one step, with purification, by the $\mathrm{H}^{-}$nucleophilic substitution of bovine milk ethanol extract, phospho oligosaccharide di-phospho asparaginyl sulfo tyrosine dipeptide.

\section{Materials and Methods}

Bovine milk ( $0.1 \mathrm{~mL}$, fat free, commercially obtained) will be placed in an Eppendorf-like $\mu$-centrifuge tube $(\sim 1.5 \mathrm{~mL}$ capacity) and ethanol $(95 \%, 1.00 \mathrm{~mL})$ will be added. The mixture will be centrifuged and the supernatant removed and the supernatant evapotated under a stream of air. To the syrup will be added $\mathrm{NH}_{4} \mathrm{OH}(1.00 \mathrm{~mL}, \mathrm{pH} 11.4,1 \mathrm{~N})$ and then $\mathrm{NaBH}_{4}(3 \mu \mathrm{L}$ of a $4 \mathrm{~N}$ solution) added. The solution will remain un-capped and allowed to stand at ambient temperature for 4 to 8 hours. The reaction mixture will then be pushed through an $\mathrm{NH}_{4}{ }^{+}$cation exchange cartridge $(\sim 1.5$
$\mathrm{mL}$, ThermoFisher, Sunnyvale, CA USA) and then the effluent pushed through a $\mathrm{Na}^{+}$form cation exchange cartridge $(\sim 1, .5 \mathrm{~mL}$, ThermoFisher, Sunnyvale, CA, USA) and the effluent frozen prior to analysis by $\mathrm{ms}$ and $\mathrm{ms}^{2}$ on a triple quadrapole mass spectrometer. $\mathrm{NaBH}_{4}$, ethanol and $\mathrm{NH}_{4} \mathrm{OH}(5 \mathrm{~N})$ will be purchased from Aldrich Chemical Company, Saint Louis, MO, USA.

The N-acetamido neuraminyl galactosyl phospho 1,5anhydro glucitol will be isolated as previously reported. [2, 10] The milk oligosaccharide dipeptide will be isolated, also, as previously reported. [2, 10]

\section{Results and Discussion}

There is supportive evidence for the formation of 2,6 anhydro $\mathrm{N}$-acetamido neuraminitol from sialylated sulfo oligosaccharide di-phospho serinyl sulfo tyrosine dipeptides. [11] Products from the PNGase-F treatment of the glycoproteins $\mathrm{K}$ casein and bovine submaxillary mucin, isolation, after having been treated with $\mathrm{NaBH}_{4}$ in $\mathrm{NH}_{4} \mathrm{OH}$ produce the anhydro monosaccharide. In each case there is evidence for a nucleophilic substitution by $\mathrm{H}^{-}$ion of the diphospho linked oligosaccharides $[11,12]$ There has been no report of such products.

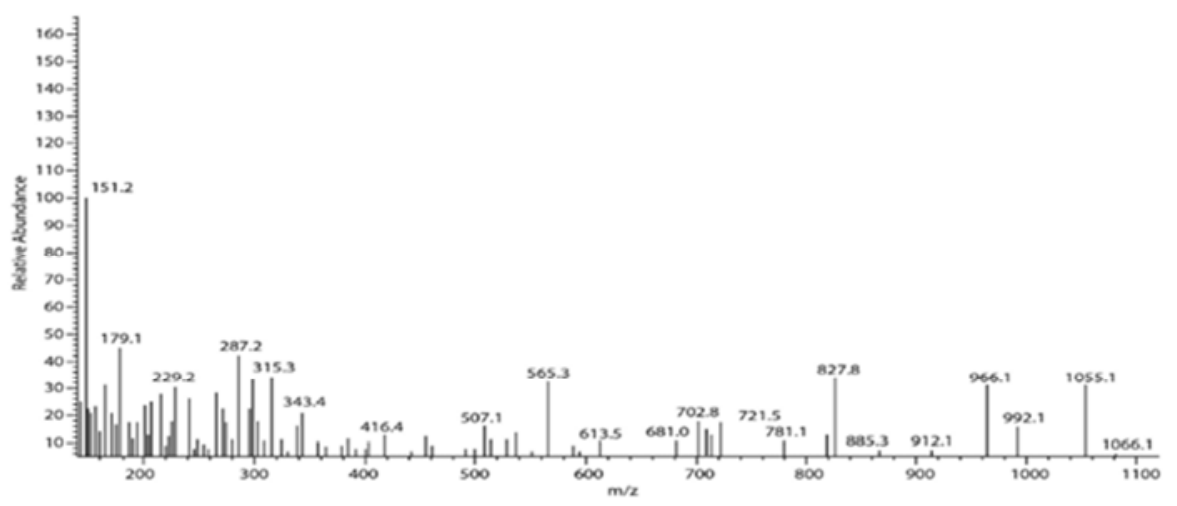

Figure 1. MS of Kappa casein after treatment with PNGase-F followed by isolation and reaction with $\mathrm{NaBH}_{4}$ in $\mathrm{NH}_{4} \mathrm{OH}$.

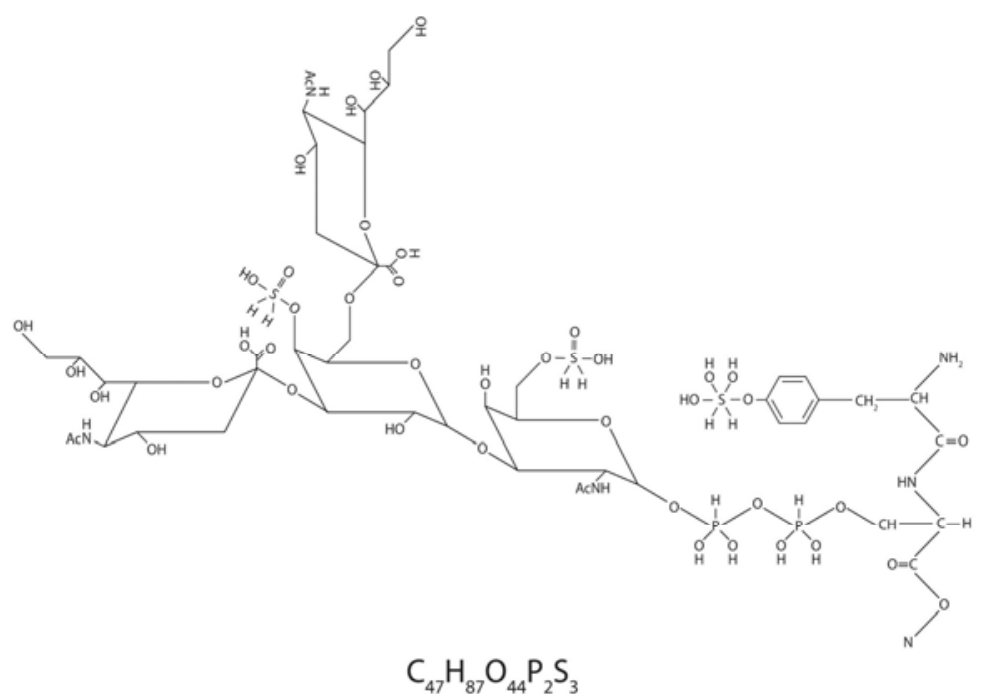

Figure 2. Denotes the structure of whole K casein O-linked oligosaccharide dipeptide resulting from the treatments described. 


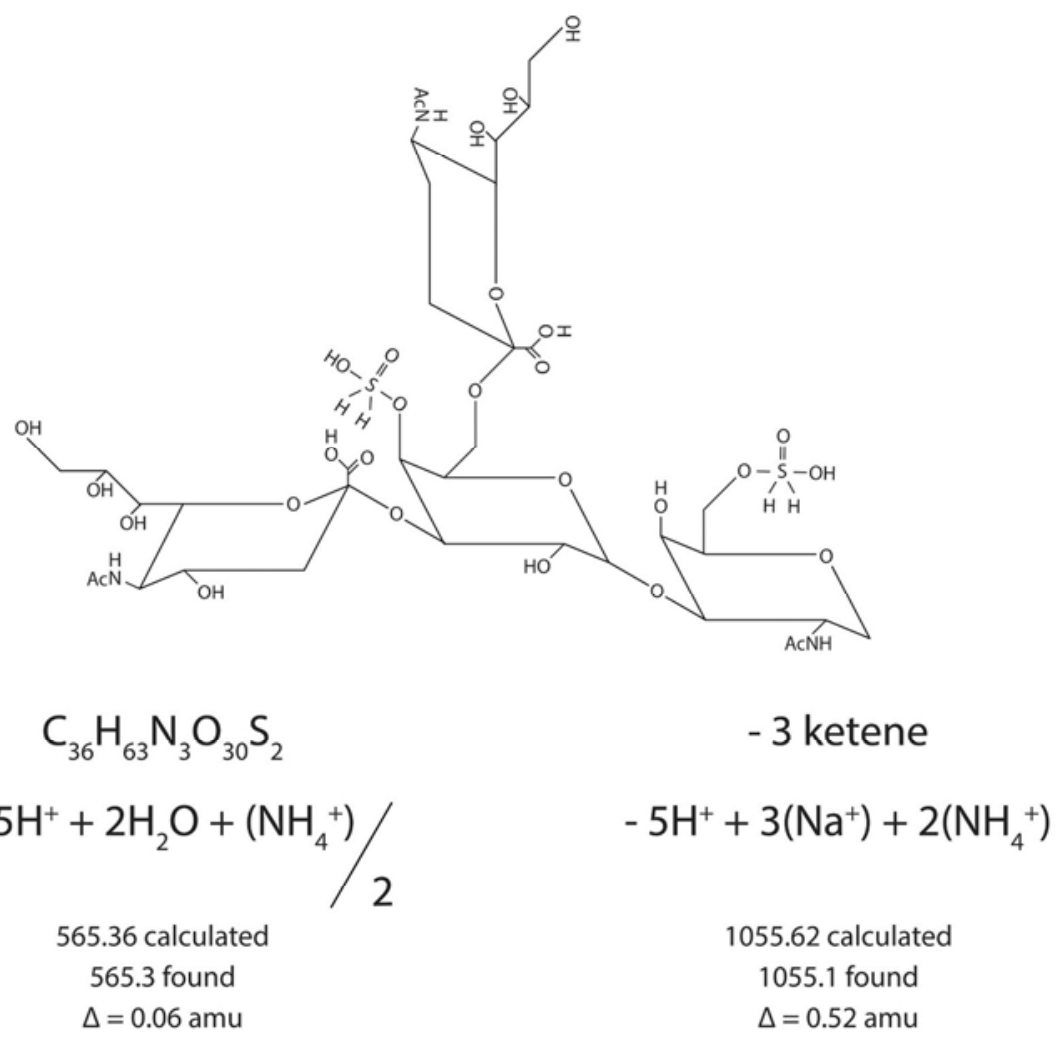

Figure 3. Depicts the structure of whole K casein O-linked oligosaccharide dipeptide. Two ions', $\mathrm{m} / \mathrm{z} 1055.1 \mathrm{and} \mathrm{m} / \mathrm{z} 565.3$ structures are shown.

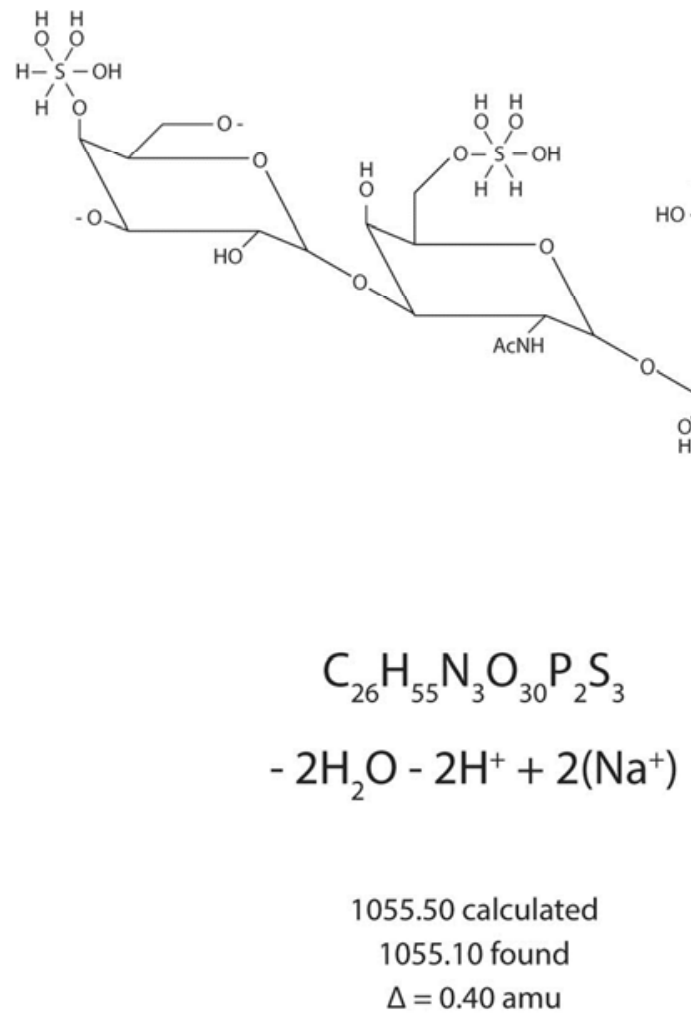

Figure 4. Describes pictorially another structure for ion, $\mathrm{m} / \mathrm{z} 1055.1$.

Conditions will be used to prepare the sialic acid derivative. The conditions for the preparation of the latter molecule will be chosen such that the 1,5 anhydro trisaccharide formation is minimized, because of the potential difficulty in separating the 2,6 anhydro neuraminitol and the 1,5 anhydro lactose or 1,5 anhydro trisaccharide. If the dipeptide remains bound to the lactosyl group, it can be removed by $\mathrm{NH}_{4}{ }^{+}$and $\mathrm{Na}^{+}$cation exchange resins. 


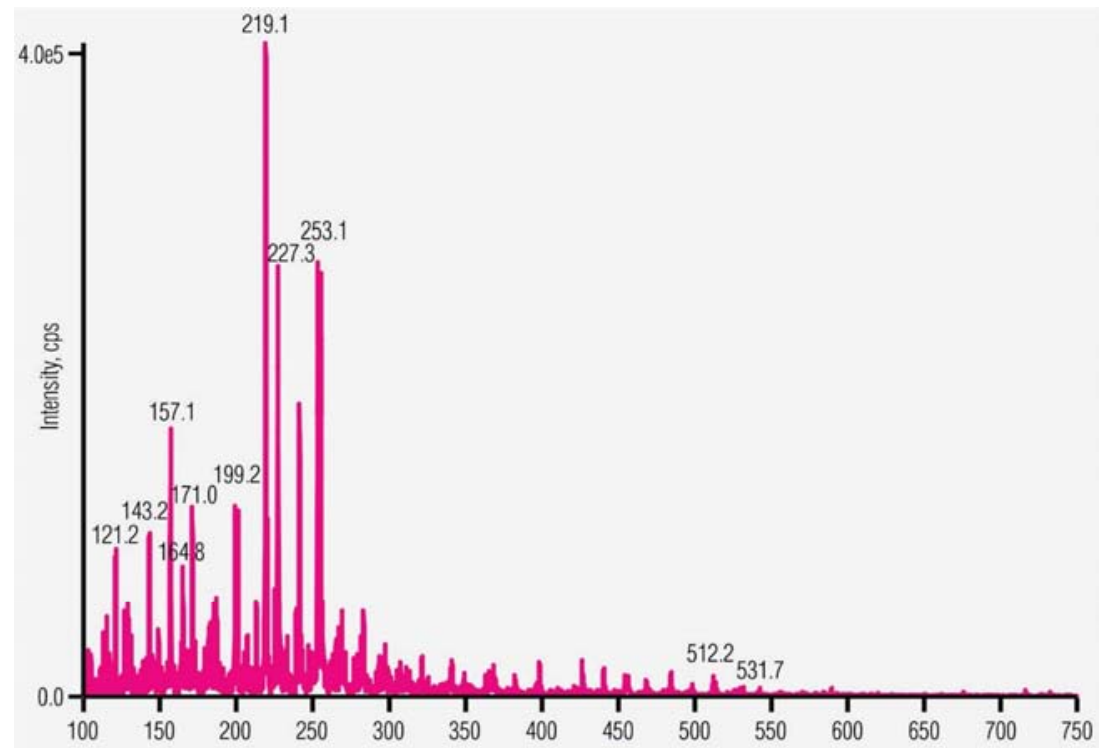

Figure 5. Mass spectrum obtained by treatment of bsm with $\mathrm{P}$ NGase-F and $\mathrm{NaBH} 4$ in $\mathrm{NH}_{4} \mathrm{OH}$.

In Figure 5 is found a mass spectrum for treatment of bsm with PNGase-F, isolation and treatment with $\mathrm{NaBH}_{4}$ in $\mathrm{NH}_{4} \mathrm{OH}$.

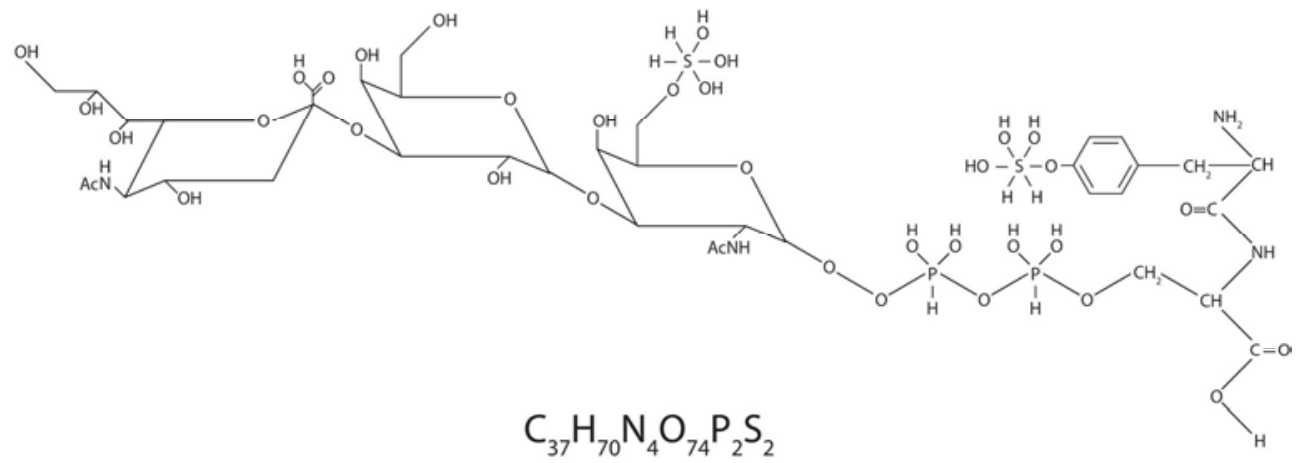

Figure 6. Shows the structure from which the bsm derived oligosaccharide dipeptide's spectrum is produced.

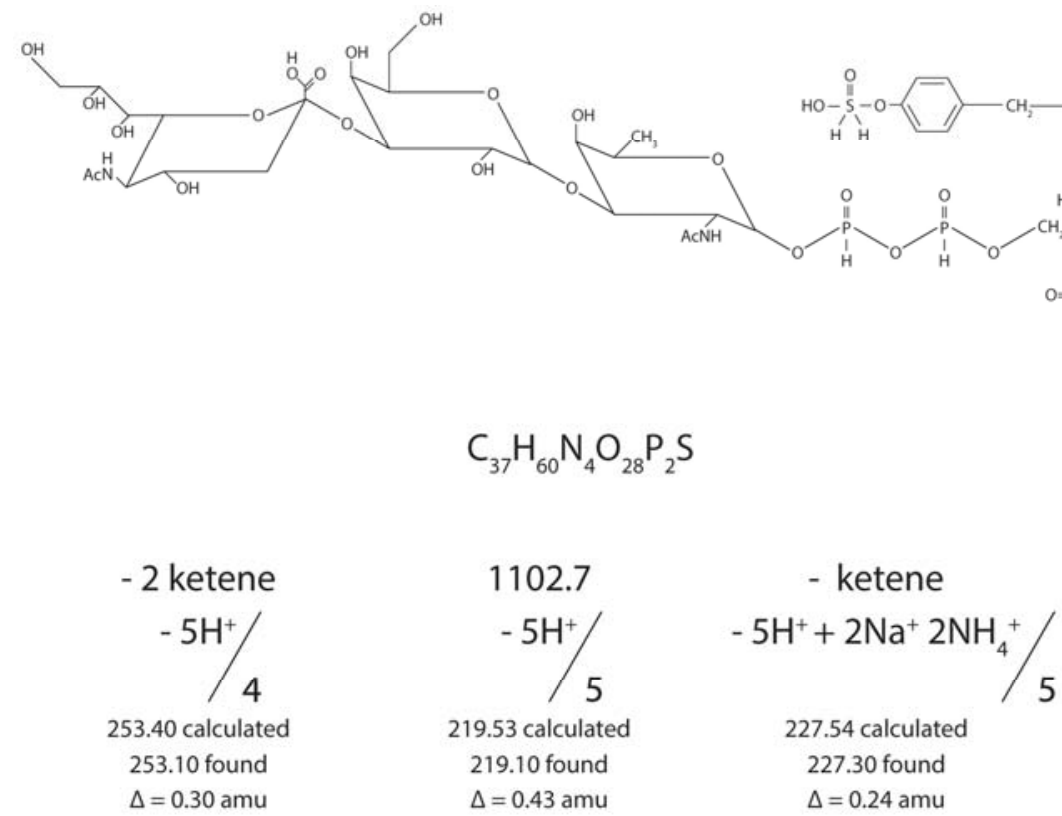

Figure 7. Ions found in mass spectrum after treatment of bsm with PNGase-F followed by $\mathrm{NaBH}_{4}$ in $\mathrm{NH}_{4} \mathrm{OH}$. 
In Figure 7 ions, m/z 253.1, m/z 219.1 (bp) and m/z 227.3 are found.

In Figure 7 is the molecule in Figure 6 except that the sulfate and phosphate deivatives are dehydrated. Also, the (di-hydrido) sulfo group on galNHAc is converted to the deoxy carbon.

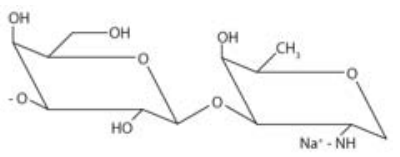

$$
\mathrm{C}_{12} \mathrm{H}_{21} \mathrm{NO}_{8}\left(\mathrm{Na}^{+}\right) /
$$

$$
\begin{gathered}
165.11 \text { calculated } \\
164.80 \text { found } \\
\Delta=0.31 \text { amu }
\end{gathered}
$$

Figure 8. In this figure is shown the structure for $\mathrm{m} / \mathrm{z} 164.8$.

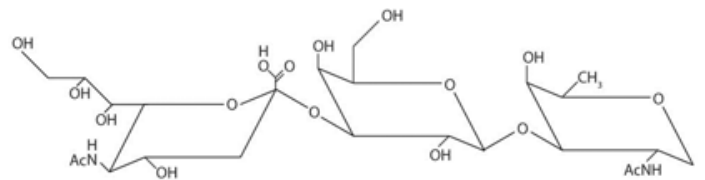

$$
\begin{gathered}
\mathrm{C}_{25} \mathrm{H}_{42} \mathrm{~N}_{2} \mathrm{O}_{17} \\
\text { - ketene } \\
-3 \mathrm{H}^{+} / 3 \\
199.14 \text { calculated } \\
199.20 \text { found } \\
\Delta=0.06 \text { amu }
\end{gathered}
$$

Figure 9. Is drawn the 1,5-anhydro trisaccharide. It is drawn with a deoxy carbon on the $\mathrm{N}$-acetamido galactosaminitol group of the trisaccharide. The ion is; $m / z 1992$.

They are produced from the drawn. Of note is the deoxy carbon at $\mathrm{C}-6$ of the $\mathrm{N}$-acetamido galactosamine. These reaction conditions may produce a deoxy group where a sulfate ester is formerly located. [13] Substitution at this monosaccharyl component with sulfate is demonstrated with this mass spectrum and its component ions and their subsequent interpretations.

Figure 8 shows a structure for ion, $\mathrm{m} / \mathrm{z}$ 164.8. This structure supports the treatise that the original O-linked oligosaccharide is substituted with sulfate at the $\mathrm{N}$-acetamido galactosamine of the oligosaccharide.

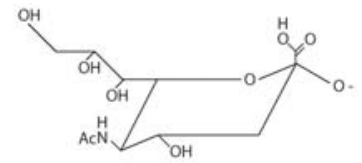

$$
\begin{gathered}
\mathrm{C}_{11} \mathrm{H}_{18} \mathrm{NO}_{9} \\
-2 \mathrm{H}^{+}+2\left(\mathrm{NH}_{4}^{+}\right) / 2 \\
171.13 \text { calculated } \\
171.0 \text { found } \\
\Delta=0.13 \text { amu }
\end{gathered}
$$

Figure 10. describes pictorially ion, $\mathrm{m} / \mathrm{z}$ 171.0.

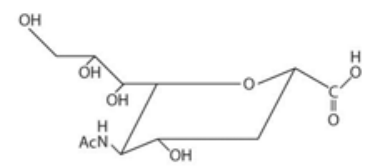

$$
\mathrm{C}_{11} \mathrm{H}_{19} \mathrm{NO}_{8}
$$

$$
\begin{array}{cc}
\text { bovine submaxillary mucin } & \text { kappa casein } \\
-2 \mathrm{H}^{+}+\mathrm{Na}^{+} / & -\mathrm{H}^{+}+\mathrm{Na}^{+} \\
157.10 \text { calculated } & \\
157.0 \text { found } & 315.19 \text { calculated } \\
\Delta=0.10 \text { amu } & 315.30 \text { found } \\
& \Delta=0.11 \text { amu }
\end{array}
$$

Figure 11. Describes the structures of the ions, $\mathrm{m} / \mathrm{z} 157.1$ from the bsm mass spectrum and ion, $m / z 315.3$ from the mass spectrum for kappa casein. It is 2,6-anhydro N-acetamido neuraminitol.

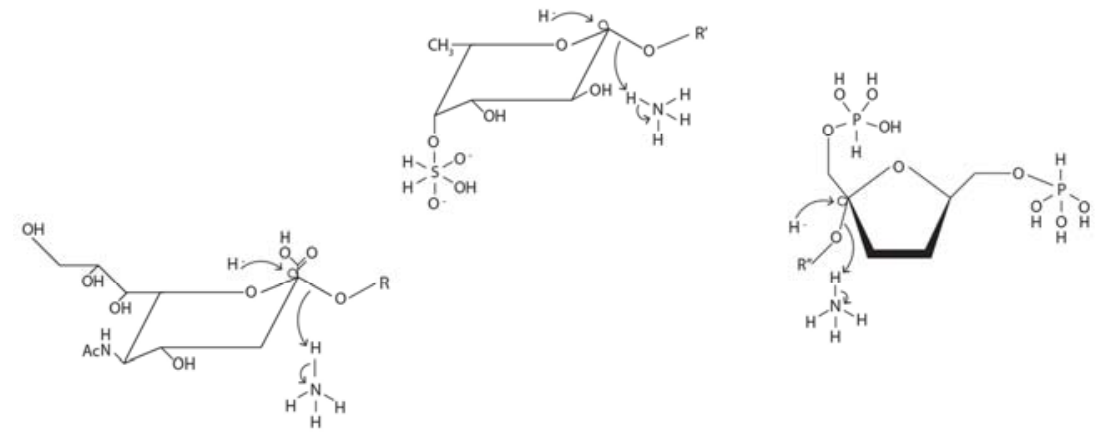

Figure 12. Products of the reaction of glycoprotein with PNGase- $\mathrm{F}$ and $\mathrm{NaBH} 4$ in $\mathrm{NH}_{4} \mathrm{OH}$. 
In Figure 12 three structures are depicted.

All three structures describe the $\mathrm{H}^{-}$nucleophilic attack on a $\mathrm{N}$-acetamido neuraminyl group, a (di-hydrido) sulfo hydrate with the L-fucosyl group and a 1,6-di-(hydrido) di-phospho di-hydrate fructo-furanosyl group. All three produce the corresponding anhydro molecule; 2,6 anhydro N-acetamido neuraminitol, 1,5-anhydro (di-hydrido) sulfo hydrate Lfucitol and di -(hydrido) di-phospho di-hydrate 2,5 anhydro mannitol (glucitol), respectively.

Bovine fetuin could be another source of sialylated 1,5 anhydro glycan or sialylated oligosaccharide di-phospho sulfo dipeptide, derivatizef or underivatized. It is a triantennary sialylated oligosaccharide di-phospho asparaginyl sulfo tyrosine dipeptide, derivative and un-derivatized [14$18]$.

\section{$-2 \mathrm{H}^{+}+2\left(\mathrm{Na}^{+}\right)$}

$$
\begin{gathered}
142.97 \text { calculated } \\
143.2 \text { found } \\
\Delta=0.23 \mathrm{amu}
\end{gathered}
$$

$$
-\mathrm{H}^{+}+\left(\mathrm{Na}^{+}\right)
$$

$$
\begin{gathered}
120.99 \text { calculated } \\
121.00 \text { found } \\
\Delta=0.01 \text { amu }
\end{gathered}
$$

Figure 13. Structures for, ions, $\mathrm{m} / \mathrm{z} 143.2$ and $\mathrm{m} / \mathrm{z} 121.1$ are drawn. The two ions are derived from the same fragment, $\mathrm{HPO}_{4}^{-}$, the singularly de-protonated $\mathrm{Na}^{+}$ salt and the doubly deprotonated ion with two $\mathrm{Na}^{+}$ions to the partially neutralized ion.

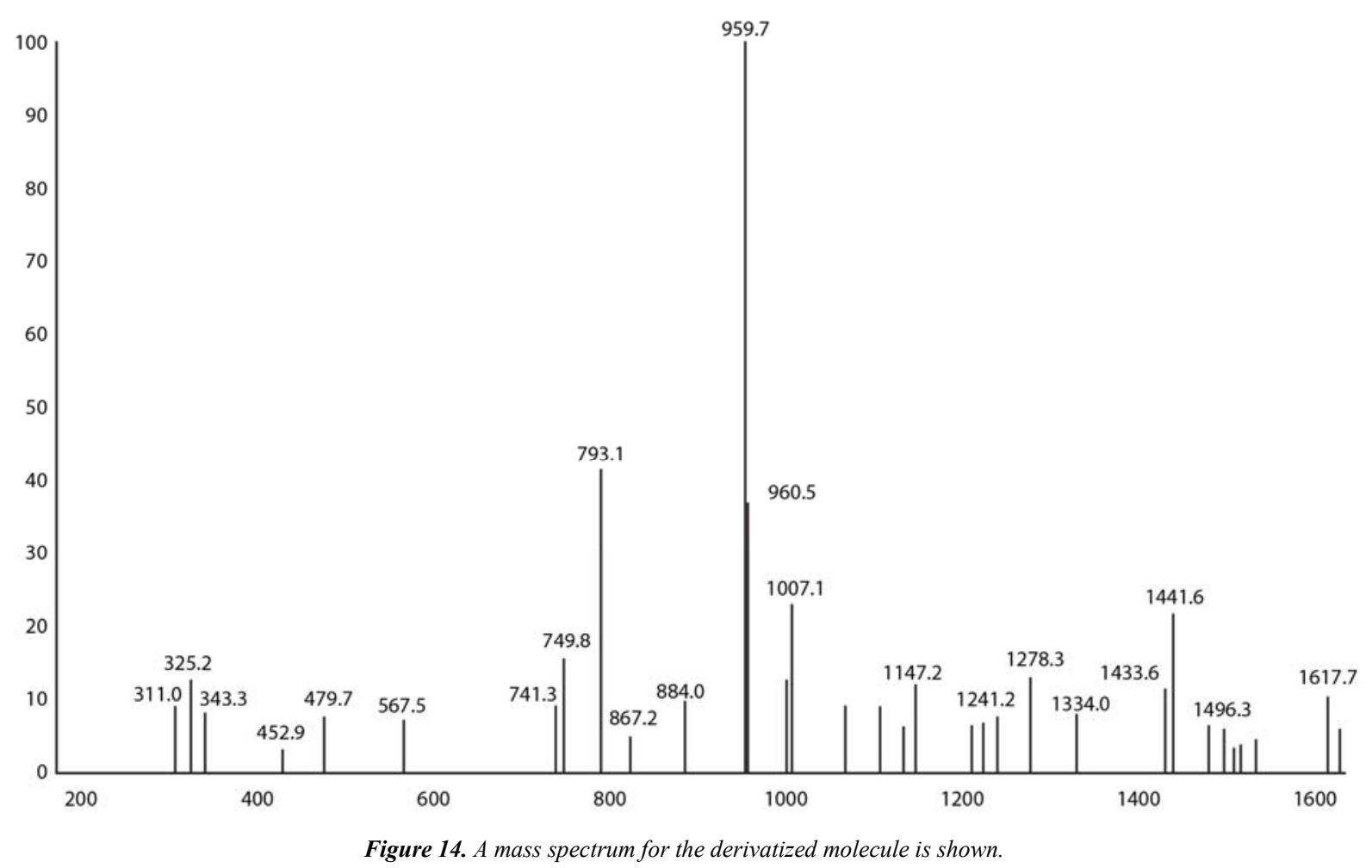

This structure shows the tri-sialyl tri-antennary glycan substituted with an L-fucosyl group on the glcNHAc one monosaccharide group removed from the 'reducing sugar.' The 'reducing sugar' glcNHAc is substituted with di-(hydrido) di-phospho di-hydrate asparaginyl (di-hydrido) sulfo hydrate tyrosine di-peptide. The L-fucosyl group or the di-phospho group rmost likely prevents PNGase-F from cleaving the asparagine to glycan linkage. grun $\mathrm{m} / \mathrm{z} 793.1$ represents the whole derivatized molecule and the ion, $\mathrm{m} / \mathrm{z} 1441.0$ represents the whole molecule less two sialyl groups. 


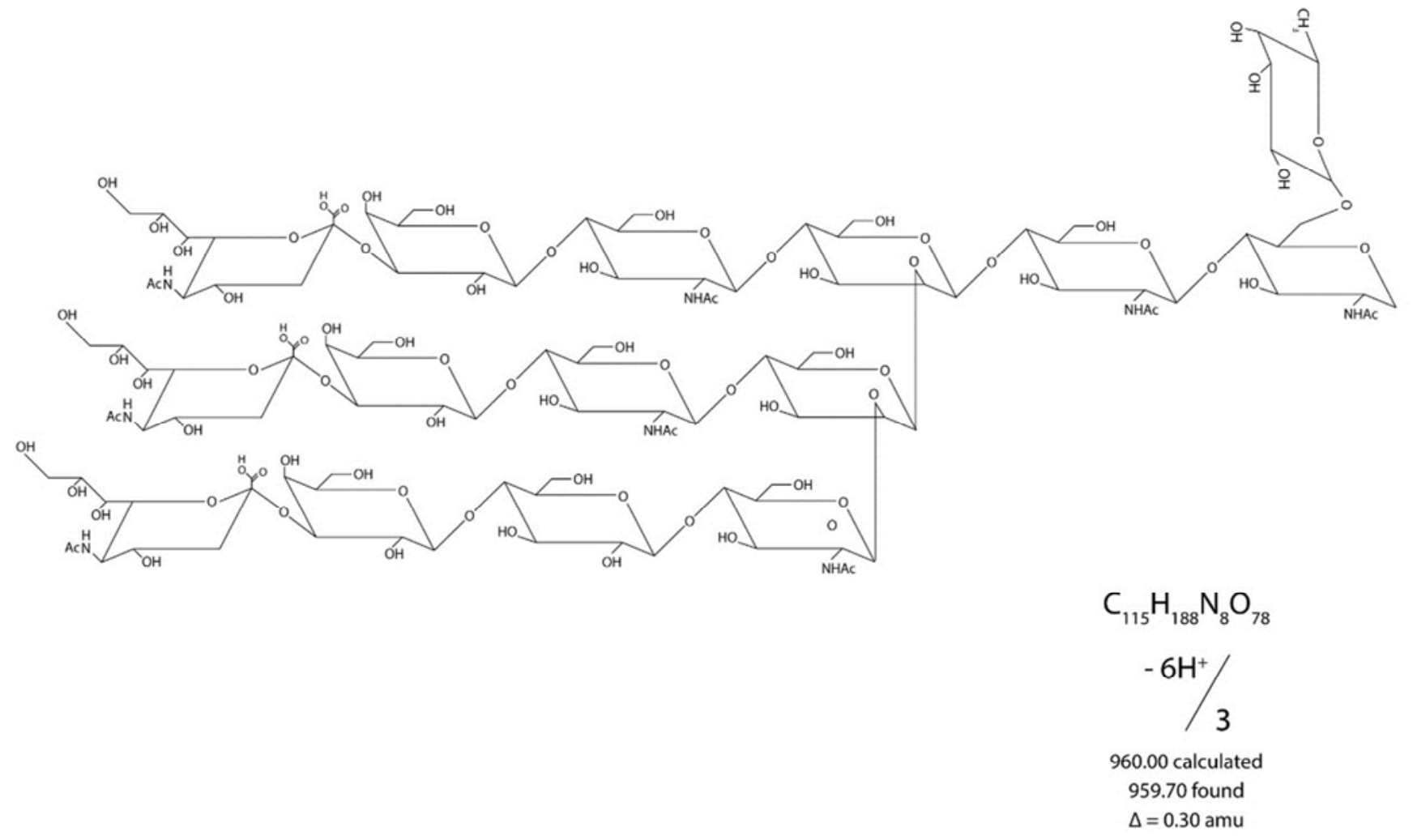

Figure 15. Ion, $\mathrm{m} / \mathrm{z}$ 959.7, is the 1,5 anhydro tri-antennary tri-sialylated glycan.

Harvey has reported the ion, $\mathrm{m} / \mathrm{z} 959$ in his work with fetuin. This molecule could also be tested for its production of 2,6 anhydro $\mathrm{N}$-acetamido neuraminitol and its inhibition of coronavirus infection.

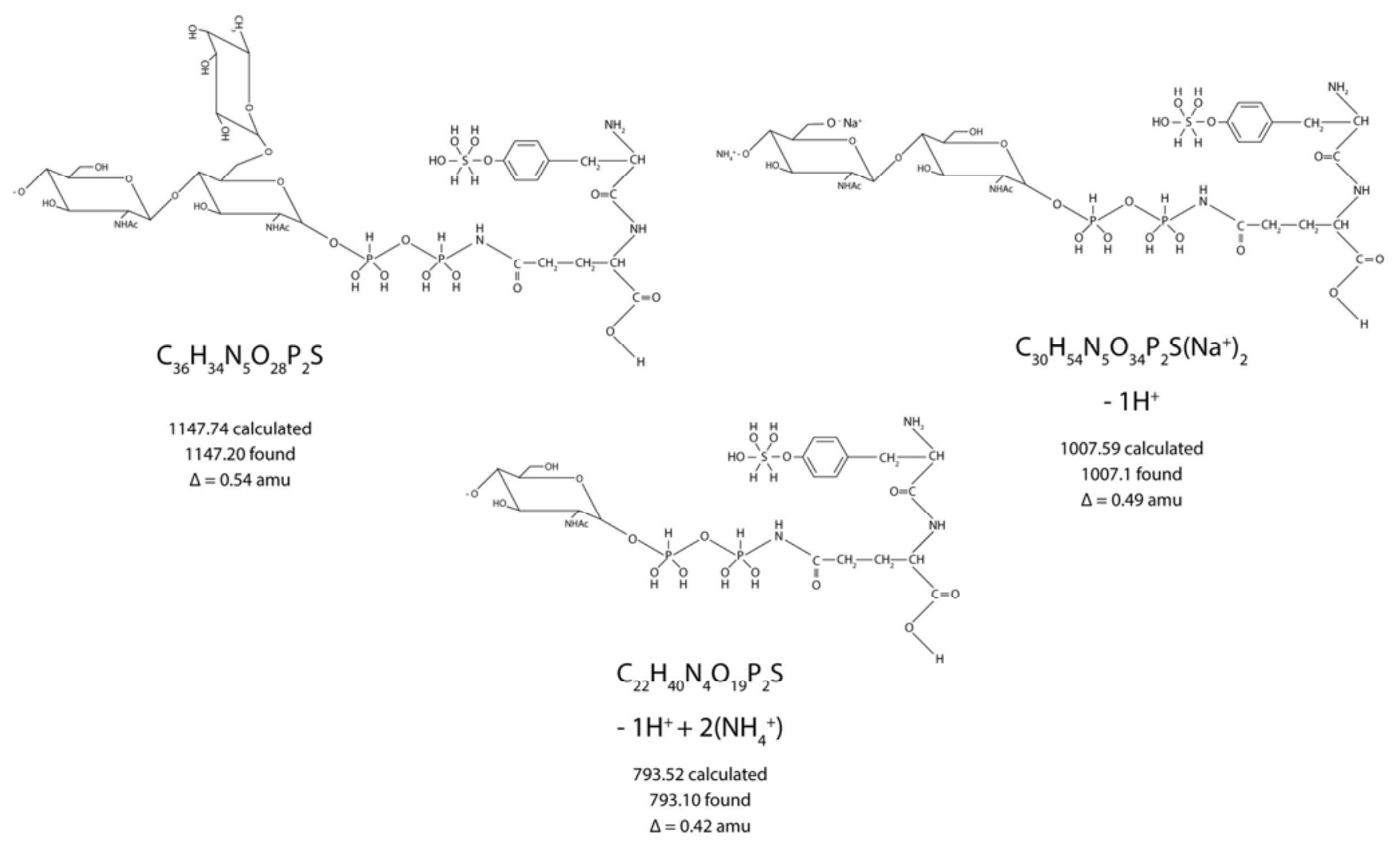

Figure 16. Ions, $\mathrm{m} / \mathrm{z}$ 1007.1, $\mathrm{m} / \mathrm{z} 1147.2$ and an alternate $\mathrm{m} /$. 


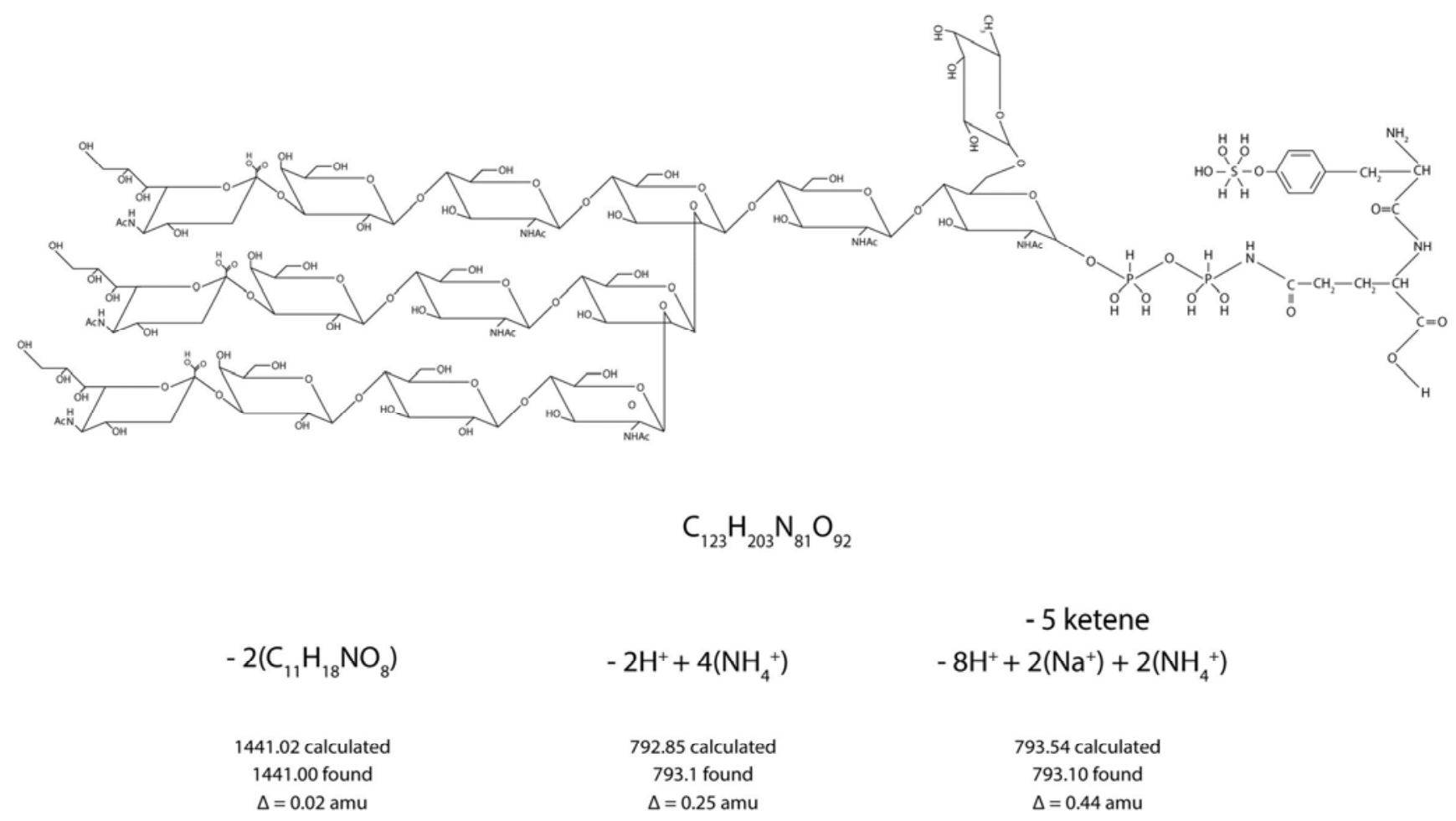

Figure 17. Ions, $\mathrm{m} / \mathrm{z}$ 1441.1, $\mathrm{m} / \mathrm{z}$ 793.1, are represented by the structures shown.

In Figure 17 are other ions in the spectrum of fetuin treated by this process.

All three ions have the same aglycone. Ion, $\mathrm{m} / \mathrm{z} 1147.2$ is drawn to show L-fucosylation of the oligosaccharide. Ion, $\mathrm{m} / \mathrm{z} 1007.1$ is drawn illustrating double substitution of the non-reducing end of the glycan. Ion, $\mathrm{m} / \mathrm{z} 793.1$, is a possible alternate structure for this molecule contributes to the relative intensity of this ion.

Kappa casein 1,5 anhydro oligosaccharide and derivatized oligosaccharide dipeptide along with bovine submaxilary mucin 1,5 anhydro oligosaccharide and fetuin 1,5 anhydro oligosaccharide and their corresponding derivatized oligosaccharide dipeptide could be tested for efficacy toward inhibiting coronavirus infection. All have terminal sialyl groups and could 'wash out' coronavirus.

A suggested method for the preparation of the title molecule would include; 4 to 8 hours at ambient temperature in an open reaction vessel. Judicious use of the $\mathrm{Na}^{+}$and $\mathrm{NH}_{4}{ }^{+}$ cation exchange resins are necessary.

In order to make the reaction of bovine milk oligosaccharide dipeptide simple to purify away from the possible rection products; 1,5-anhydro lactose, 1,5-anhydro glucose, the reaction conditions must prevent the cleavage of glycosyl-di-(hydrido) di-phospho group. A search for such conditions would include the variables; capped versus uncapped reaction vessel, time of the reaction, and ambient temperature or below $0^{\circ} \mathrm{C}$. In the case of bovine milk, a nearly pure 1,5-anhydro trisaccharide was obtained when the reaction vessel was closed, to keep $\mathrm{pH}$ high, at ambient temperature for 2 hours reaction time. [2] Clearly these conditions must be avoided. 1,5-anhydro di-(di-hydrido)-di-sulfo $\mathrm{K}$ casein tetrasaccharide is produced. [11] This was also obtained by closing the reaction vessel for 8 hours at ambient temperature. In Figures 8 and 9 there are ions, m/z 164.8 and $\mathrm{m} / \mathrm{z} 199.2$, that show the 1,5 anhydro deoxy molecule. The reaction conditions are; 12 hours at ambient temperature in a closed reaction vessel. Reaction conditions here, to begin, could be 4 to 8 hours at ambient temperature in an open reaction vessel. With an open reaction vessel, $\mathrm{NH}_{3}(\mathrm{~g})$ is released to the air, and the $\mathrm{pH}$ drops. This prevents the deprotonation of $\mathrm{R}-\mathrm{P}-\mathrm{OH}$ and $\mathrm{R}-\mathrm{S}-\mathrm{OH}$. It prevents the formation of the $\mathrm{P}=\mathrm{O}$ and $\mathrm{S}=\mathrm{O}$ moeities and thus the weakening of the $\mathrm{R}-\mathrm{CH}_{2}-\mathrm{O}$ bond, the alcohol substituted to $\mathrm{O}$ atom bond, and the R-CH-O-P (O-R'), anomeric $\mathrm{C}-1$ to $\mathrm{O}-1$ bond. The $\mathrm{H}^{-}$nucleophile can then attack C-1 or C-6 atoms. [2, 5, 6, 12] Therefore, the lowered $\mathrm{pH}$ of the reaction mixture, allows the reaction product lactosyl-di-(hydrido) di-phospho di-hydrate asparaginyl (di-hydrido) sulfo hydrate tyrosine to be removed from the reaction mixture by pushing the reaction mixture through $\mathrm{NH}_{4}{ }^{+}$and $\mathrm{Na}^{+}$form cation exchange resins. The effluent should contain only the 2,6-anhydro Nacetamido neuraminitlol. Conditions may be used to obtain the 1,5 anhydro oligosaccharides from $\mathrm{K}$ casein, bovine submaxillary mucin, fetuin and bovine milk.

\section{Acknowledgements}

Author would like to acknowledge the efforts of Beth Bachman Caufield for her moral support.

Ricardo Samiento for his love of the Word of God and 
continued support and Jeff Alvestad for his weekly gifts to Saint Andrew's Lutheran Church all are annointed men and woman of God as good shepherds. Thanks also must go to Isaac Woolery of Ames Copyworks for his excellent graphic design work in this manuscript.

\section{References}

[1] Schultze, B.; Cavanaugh, D.; Heirrer; Neuraminidase treatment of avian infectious bronchitis coronavirus reveals a hemagglutinating activity that is dependent on sialic acid containing receptors on erythrocytes Virology 189 (2) 792-794 (1992).

[2] Christus, Madson, M.; Possible treatment of Mycobacterium lepramatous with bovine milk World journal of food science and technology 2 (3) 55-61 (2018).

[3] Vlaska, R.; Luytjes, W.; Spaan, W.; Palese, P.; Human and bovine coronaviruses recognise sialic acid containing receptors similar to those of influenza $\mathrm{C}$ virus Proceedings of the national academy of.

[4] Christus, J.; Madson, M.; Possible treatment of Mycobacterium lepramatous with bovine milk World journal of food science and technology 2 (3) 55-61 (2018).

[5] Christus, J.; Madson, M.; Preparation of possible P selectin inhibitor from bovine thyroglobulin (di-hydrido) sulfo hydrate. 1,5-anhydro L-fucitol World journal of food sci.

[6] Christus, J.; Madson, M.; Preparation of 2,5-anhydro di(hydrido) di-phosphate di-hydrate mannitol (glucitol) from banana fruit yields a possible fructose 1,6 di-phosphate aldolase inhibitor World journal of food science and technology accepted for publication (2019).

[7] Christus, J.; Madson, M.; Determination sulfate monosaccharide substitution World journal of food science and technology accepted for publication (2020).

[8] Mane, R.; Goch, S.; Singh, S.; Chopale, B.; Dhavale, D.; Synthesis and anomeric 1,5-anhydro sugars as conformatinally locked selective alpha mann osidase inhibitors Bioorganic and medicinal chemistry 19 (22) 6720-6725 (20y11).
[9] Shively, J.; Conrad, H.; Formation of anhydro sugars in the chemical depolymerization of heparin Biochemistry 15 (18) 3932-3942 (1976).

[10] Christus, J.; Madson, M.; Determination of anomeric configurations of Kappa casein O-linked oligosaccharide dipeptide World journal of food science and technology accepted for publication (2020).

[11] Niwa, T.; Tsuruoka, T.; Goi, H.; Kodama, Y.; Itoh, J.; Inouye, S.; Yamada, Y.; Niida, T.; Nobe, M.; Ogawa, Y.; Novel glycosidase inhibitors $\mathrm{B}$ and $\mathrm{D}$-mannonic-delta-lactam The journal of antibiotics 37 (12) 1579-1586 (1984).

[12] Christus, J.; Madson, M.; Possible Mimics of Duffy Binding Protein-II for Plasmodium vivax Binding Endothelial Cells or Binding Plasmodium falciparum by Mimicking Epitope on Erythrocyte Binding Antigen-175 World journal of food science and technology 2 (2) 44-54 (2018).

[13] Madson, M.; Metho d of discerning substitution of carbohydrate esters patent US 9,726,671 B2 (2017).

[14] Harvey, D.; Fragmentation of negative ions from carbohydrates: part 1 . Use of nitrate and other anionic adducts for the production of negative ion electrospray spectrum. Journal of American society of mass spectrometry 16 (5) 622$630(2005)$.

[15] Schwegman-Wessels, C.; Herrler, G.; Sialic acid as a r Krempl, C.; Schultze, B.; Laude, H.; Herrier, G.; Point mutation in the $\mathrm{S}$ protein connect the sialic acid binding activity with the enteropatogenicity of transmissible gastroenteritis coronavirus Journal of virology 71 (4) 3285-3287 (1997).

[16] Valcadlo, D.; Davies, G.; Mechanistic insights into glycosidase chemistry Current opinions in chemical biology 12 (5) 539-555 (2008).

[17] Schwegman-Wessels, C.; Herrler, G.; Sialic acid as a receptor determinant for coronavirus Glycoconjugate journal 23 (1-2) 51-58 (2006).

[18] Holmes, K.; SARS-associated coronavirus New england journal of medicine 348 (20) 1948-1951 (2003). 